\title{
Erratum to: Optimum CT reconstruction parameters for vascular and hepatocellular carcinoma models in a liver phantom with multi-level dynamic computed tomography with 64 detector rows: a basic study
}

\author{
Kazuhiro Murotani • Nobuyuki Kawai • Morio Sato • \\ Hiroki Minamiguchi • Motoki Nakai · Tetsuo Sonomura • \\ Seiki Hosokawa · Tadayoshi Nishioku \\ Published online: 30 March 2013 \\ (C) Japanese Society of Radiological Technology and Japan Society of Medical Physics 2013
}

Erratum to: Radiol Phys Technol

DOI 10.1007/s12194-013-0203-8

The correct name of the first author should be given as Kazuhiro Murotani, and not Murotani Kazuhiro as in the original publication.

The online version of the original article can be found under doi: 10.1007/s12194-013-0203-8.

K. Murotani · N. Kawai · M. Sato $(\bowtie) \cdot$ H. Minamiguchi · M. Nakai · T. Sonomura $\cdot$ S. Hosokawa $\cdot$ T. Nishioku Department of Radiology, Wakayama Medical University, 811-1 Kimiidera, Wakayama, Wakayama 641-8510, Japan e-mail: morisato@wakayama-med.ac.jp 